\title{
Some new theoretical and computational results around the Jacobian conjecture
}

\author{
Tuyen Trung Truong \\ University of Oslo, 0316 Oslo, Norway \\ tuyentt@math.uio.no
}

\begin{abstract}
In this paper, we study a so-called Condition $\mathrm{C} 1$ on square matrices with complex coefficients and a weaker Condition C2. For Druzkowski maps Condition C2 is equivalent to the Jacobian conjecture. We show that these conditions satisfy many good properties, in particular are satisfied by a dense subset of the set of square matrices of a given rank $r$. Based on this, we propose a heuristic argument for the truth of the Jacobian conjecture. We propose some new equivalent formulations and some generalisations of the Jacobian conjecture, and some approaches (including computer algebra and numerical methods) toward resolving it. We show that some of these equivalent formulations are automatically satisfied by generic Druzkowski matrices. Applications and experimental results are included.

Keywords: Druzkowski maps; Groebner basis; Hilbert's Nullstellensatz; Jacobian conjecture

Mathematics Subject Classification 2010: 13B25, 14Qxx, 14Rxx, 15Bxx
\end{abstract}

\section{Introduction}

\subsection{A heuristic argument for the truth of the Jacobian conjecture}

The famous Jacobian Conjecture is the following statement:

Jacobian Conjecture. Let $F=\left(F_{1}, \ldots, F_{n}\right): \mathbb{C}^{n} \rightarrow \mathbb{C}^{n}$ be a polynomial map such that $J F$ (the Jacobian matrix $\left.\left(\partial F_{i} / \partial x_{j}\right)_{1 \leq i, j \leq n}\right)$ is invertible at every point. Then, $F$ has a polynomial inverse.

The Jacobian conjecture was first stated by Keller in 1939. Polynomial maps with invertible Jacobian matrices are called Keller maps. We denote by $J C(n)$ the Jacobian Conjecture in dimension $n$, and by $J C(\infty)$ the statement that $J C(n)$ holds for every $n$. In the literature $J C(\infty)$ is usually called the generalized Jacobian Conjecture. This conjecture has attracted a lot of works, and many partial results were found. For example, Magnus - Applegate -Onishi -Nagata proved $J C(2)$ for $F=(P, Q)$ where the GCD of the degrees of $P, Q$ is either a prime number or $\leq 8$; Moh proved $J C(2)$ for $\operatorname{deg}(F) \leq 100$; Wang proved that $J C(n)$ holds if $\operatorname{deg}(F)=2$; and $\mathrm{Yu}$ (also Chau and Nga) proved that if $F(X)-X$ has no linear term and has all non-positive coefficients then JC holds for $F$. For more details the readers can consult the reference list and the references therein. An excellent survey is the book [19]. We note that the $\mathbb{R}$-analog of $J C(2)$ (in this case, we require only that 
the map $F$ is bijective, since its inverse may not be a polynomial as the example $F(x)=x+x^{3}$ shows) is false, by the work of Pinchuk (Section 10 in [19]).

There have been many reductions of the generalised Jacobian Conjecture $J C(\infty)$. One of these reductions is due to Bass, Connell, Wright and Yagzhev, who showed that to prove $J C(\infty)$, it is enough to prove for all $F(x)=x+H(x)$ and all $n$, where $H(x)$ is a homogeneous polynomial of degree 3 (Section 6.3 in [19]). Druzkowski made a further simplification (Section 6.3 in [19])

Theorem 1.1 (Druzkowski). $J C(\infty)$ is true if it is true for all the maps $F$ of the form $F(x)=\left(x_{1}+l_{1}(x)^{3}, \ldots, x_{n}+l_{n}(x)^{3}\right)$ with invertible Jacobian JF, here $l_{1}, \ldots, l_{n}$ are linear forms.

Later, Druzkowski [14] simplified even further showing that it is enough to show for the above maps with the additional condition that $A^{2}=0$, where $A$ is the $n \times n$ matrix whose $i$-th row is $l_{i}$. We then simply say that a matrix is Druzkowski if the corresponding map $F_{A}(x)=\left(x_{1}+l_{1}(x)^{3}, \ldots, x_{n}+l_{n}(x)^{3}\right)$ is Keller, i.e. the determinant of its Jacobian is 1.

A lot of efforts have been devoted to showing that the Druzkowski maps are polynomial automorphisms (Section 7.1 in [19] and for recent developments see [6-8], and for a comprehensive reference on this topic see [4]). There are many partial results proved for this class of maps, for example it is known from the works of Druzkowski, Hubbers, Yan and many other people that the Druzkowski maps are polynomial automorphisms if either the rank of $A$ is $\leq 4$ or the corank of $A$ is $\leq 3$. In particular, the Jacobian conjecture was completely checked for Druzkowski maps in dimensions $\leq 8$ (see [4], also for stronger properties that can be proved for these polynomial automorphisms). Some new results on Druzkowski maps in dimension 9 have been obtained recently in [30] and [6-8]. A common theme of these proofs is that in these cases the Druzkowski maps are "equivalent" to some other polynomial maps for which the Jacobian conjecture is previously known to be true. It is not easy to see whether this strategy can work for higher ranks or coranks.

Despite these encouraging results, there has been little progress on checking the Jacobian conjecture for Druzkowski maps in higher dimensions. One of the difficulties for this lies in the fact that the structure of the Druzkowski maps is not well-understood, in the sense that for a large enough $n$ there is no easy way to produce all $n \times n$ Druzkowski matrices (for small values $n=3,4,5$, there are classifications by Meisters, Wright and Hubbers, see [19] and [22]). Because of this, even if we have already verified the Jacobian conjecture for the Druzkowski maps up to a certain dimension, say $n=10$, it is still hard to decide whether the Jacobian conjecture will hold for these maps in higher dimensions. A striking illustration of this undecidability is the following fact proved by Rusek [26], that the set of $n \times n$ Druzkowski matrices is not irreducible for $n \geq 530$. This result implies that an obvious geometric approach to $J C(\infty)$, based on the irreducible of the set of Druzkowski matrices of a given dimension $n$, does not work. However, we will later show that $J C(\infty)$ is equivalent to a geometric condition based on connectedness of 
certain varieties related to Druzkowski matrices.

The main purpose of this paper is to propose some conditions (namely, Conditions $\mathrm{C} 1$ and $\mathrm{C} 2$ below) which are more amenable to using computer programs to check - on Druzkowski matrices - than conditions currently used in checking the Jacobian conjecture. This is justified by that these conditions concern smaller systems of polynomials, and that they concern only the injectivity of a map - which is weaker than the bijectivity of a map. (See Section 5 for more detail on this.) The validity of these conditions for either a generic Druzkowski matrix or for all Druzkowski matrices with integer coefficients will prove the Jacobian conjecture. We also show, theoretically and via examples, that these conditions make the computations needed for the direct approach (that of showing Druzkowski maps are injective) a lot faster. Contrast to the case of dimension 2, we note that the truth of the Jacobian conjecture in higher dimensions is not universally believed, the main reason is because of lack of enough evidence (for example the paper [17] reported that the majority of participants of a conference in 1997 voted that the Jacobian conjecture is false). We hope that this paper will give some more support to the opposite conclusion and illustrate the idea that computers may be used in seeking of a solution to the Jacobian conjecture $J C(\infty)$. More precisely, the idea is to investigate the small dimensions using computer programs and then make an inductive guess.

Heuristic argument for the truth of the Jacobian conjecture. In Theorem 1.5, we will show that Conditions $\mathrm{C} 1$ and $\mathrm{C} 2$ below hold for many square matrices, in particular for a dense set of all square matrices. Hence it is very reasonable that they are also true for a dense set of Druzkowski matrices, and the latter claim is enough for the truth of the Jacobian conjecture (see below). Another evidence for this heuristic argument is Theorem 1.8 below.

Remark on the effectiveness (time-saving) of our approaches. In Theorem 1.8 we will show that a generic Druzkowski matrix satisfies the Conjecture NC below. In other words, this equivalent formulation of the Jacobian conjecture holds with the probability of 100 percent. Based on this, we explain in Section 5.1 that we expect to be able to check whether a given Druzkowski matrix satisfies these criteria very quickly. This is a sign that our approaches seem very promising.

The arguments in this paper are based, besides the Druzkowski reduction and Theorem 1.7 and Lemma 3.1 to be stated below, on two other results on polynomial automorphisms. The first of these results belongs to Connell and van den Dries (Proposition 1.1.19 in [19]) :

Proposition 1.1. If for some $n \geq 2$ there is a counter example to $J C(n)$ then there is a counter example to $J C(N)$ (for some $N>n$ ) with integer coefficients.

Applying this proposition, in order to prove the Jacobian conjecture for every dimensions, it suffices to do so for polynomials with integer coefficients. From the proof of the reductions by Bass, Connell, Wright, Yagzhev and Druzkowski ( [19]), it is clear that we need to consider only Druzkowski matrices with integer coefficients. 
The second of the results mentioned above is an invertibility criterion for polynomial maps using derivations (Section 3.1 in [19]). Here we briefly recall this criterion. Let $F=\left(F_{1}, \ldots, F_{n}\right): \mathbb{C}^{n} \rightarrow \mathbb{C}^{n}$ be a polynomial map such that $F(0)=0$ and $\operatorname{det}(J F)=1$. We then define related derivations by the formula

$$
\left(\frac{\partial}{\partial F_{1}}, \ldots, \frac{\partial}{\partial F_{n}}\right)^{t}:=\left((J F)^{-1}\right)^{t}\left(\frac{\partial}{\partial x_{1}}, \ldots, \frac{\partial}{\partial x_{n}}\right)^{t} .
$$

(Here $(.)^{t}$ means the transpose of a matrix.) Now we add $n$ new variables $y_{1}, \ldots, y_{n}$ and consider the derivation

$$
D_{F}:=y_{1} \frac{\partial}{\partial F_{1}}+\ldots+y_{n} \frac{\partial}{\partial F_{n}} .
$$

Let $d=\operatorname{deg}(F)$ and put $N=d^{n-1}+1$. We have (Proposition 3.1.4 in [19])

Proposition 1.2. $F$ has a polynomial inverse if and only if $D_{F}^{N} x_{i}=0$ for all $i=1, \ldots, n$.

Applying this proposition, we see that to prove the Jacobian conjecture in every dimensions, it suffices to do so for a dense set of Druzkowski maps. We summarise these as the following

Theorem 1.2. For the Jacobian conjecture to hold in every dimensions, it suffices to either

i) Show that all Druzkowski maps whose matrix has integral coefficients satisfy the Jacobian conjecture,

or

ii) Show that a dense set of Druzkowski maps satisfies the Jacobian conjecture.

Remarks. While the reduction to integer coefficients have been studied quite extensively (e.g. Section 10.3 in [19], and also for the related topic of Jacobian conjecture in positive characteristics), it seems that the reduction to a dense set of maps has not been widely discussed. (Note that in [9], a good dense subset of all real polynomial maps whose Jacobian is invertible everywhere was identified. However, for polynomials on the field of real numbers, the density property does not work.)

\subsection{Main results in this paper}

Let us fix some notations to be used throughout the paper.

Notations. We will use the following notations. For vectors $u, v \in \mathbb{C}^{n}$, we define $u * v:=\left(u_{1} v_{1}, \ldots, u_{n} v_{n}\right)$ (coordinate-wise multiplication) and $u^{k}=u * u * \ldots * u$ ( $k$-th self-multiplication of $u$ ), and we define by $\Delta[u]$ the diagonal $n \times n$ matrix whose $(i, i)$-th entry is $u_{i}$. Thus, the Druzkowski maps and their Jacobians can be written as (here $x \in \mathbb{C}^{n}$ is viewed as a column vector)

$$
\begin{aligned}
F(x) & =x+(A \cdot x)^{3}, \\
J F(x) & =I d+3 \Delta\left[(A \cdot x)^{2}\right] . A .
\end{aligned}
$$


For a fix $n$, we let $\mathcal{M}_{n}$ denote the set of all $n \times n$ matrices with coefficients in $\mathbb{C}$. We also use the following notations:

$$
\begin{aligned}
V_{n}:= & \left\{(y, z, A) \in \mathbb{C}^{n} \times \mathbb{C}^{n} \times \mathcal{M}_{n}: \operatorname{det}\left(I d+\Delta\left[(s z+t y)^{2}\right] \cdot A\right)=1 \forall s, t \in \mathbb{C},\right. \\
& \left.z+A \cdot\left(z^{3}+z * y^{2}\right)=0\right\}, \\
W_{n}:= & \left\{(y, z, A) \in \mathbb{C}^{n} \times \mathbb{C}^{n} \times \mathcal{M}_{n}: \operatorname{det}\left(I d+\Delta\left[(s z+t A \cdot y)^{2}\right] \cdot A\right)=1 \forall s, t \in \mathbb{C},\right. \\
& \left.z+A \cdot\left(z^{3}+z *(A \cdot y)^{2}\right)=0\right\} .
\end{aligned}
$$

Note that while these two varieties look very similar, they are different in that in $V_{n}$ only $y$ appears in the equations and in $W_{n}$ only $A . y$ appears in the equations. Note also that for a Druzkowski matrix $A$, then in $W_{n}$ we do not need to check the condition $\operatorname{det}\left(I d+\Delta\left[(s z+t A \cdot y)^{2}\right] \cdot A\right)=1$

The starting point of our work is the following, to be derived in Section 3.

Theorem 1.3. Let $A$ be an $n \times n$ matrix and $F_{A}(x)=x+(A . x)^{3}: \mathbb{C}^{n} \rightarrow \mathbb{C}^{n}$ the corresponding cubic linear map. Then $F_{A}$ is an automorphism if and only if for every $y$, the solutions to $z+A .\left(z^{3}\right)+A \cdot\left(z *(A . y)^{2}\right)=0$ are exactly $z=0$.

Based on Theorem 1.3, we are now ready to state the two conditions.

Condition C1. An $n \times n$ matrix $A$ satisfies Condition C1 if whenever $(y, z, A) \in V_{n}$ then $z=0$.

Condition C2. An $n \times n$ matrix $A$ satisfies Condition C2 if whenever $(y, z, A) \in W_{n}$ then $z=0$.

As a first application of the new formulation of the injectivity of a Druzkowski map in Theorem 1.3, we give a simple and direct proof of the following result, which generalises the well-known result ( [5] and [13]) that a Druzkowski map whose Jacobian is symmetric satisfies the Jacobian conjecture (the results in these papers correspond to the case $J=I d$ and $B=A^{t}$ is the transpose of $A$ in the next theorem). The same proof can be applied to some similar situations.

Theorem 1.4. Condition $C 2$ holds for an $n \times n$ matrix $A$ if there is a matrix $B$ and a diagonal matrix $J$, such that for all $x \in \mathbb{C}^{n}$ we have $\Delta\left[(A x)^{2}\right] . A=B . \Delta\left[(A x)^{2}\right] . J$.

Condition C2 is clearly weaker than Condition C1, and for Druzkowski matrices it will be seen later that Condition $\mathrm{C} 2$ and the Jacobian conjecture are equivalent. Let $D$ be an invertible diagonal $n \times n$ matrix. It can be easily checked that if $(y, z, A) \in V_{n}$ then $\left(D y, D z, D A D^{-3}\right) \in V_{n}$, and if $(y, z, A) \in W_{n}$ then $\left(D^{3} y, D z, D A D^{-3}\right) \in W_{n}$. In particular, if $A$ is a Druzkowski matrix, then $D A D^{-3}$ is also a Druzkowski matrix. More generally, it can also be checked that a matrix $A$ satisfies Condition $\mathrm{C} 2$ iff any matrix $B$ cubic-similar to it satisfies Condition C2 (for definition and some properties of cubic-similarity, see [25]).

Condition $\mathrm{C} 1$ holds in the cases when $A$ has small sizes $(n=2,3)$ but it does not hold for $n=4$ and bigger (see Section 2). In contrast, as stated in Section 3, we conjecture that Condition $\mathrm{C} 1$ holds for all Druzkowski matrices. By standard 
arguments, it is easy to check that Condition $\mathrm{C} 1$ holds in the following cases: i) $A$ has rank 1; ii) $A$ is an upper or lower triangular matrix; and iii) all the principal minors of $A$ are non-zero. In particular, Condition C1 holds for a generic $n \times n$ matrix. This is in line with the heuristic argument for the truth of $J C(\infty)$ which we presented above. The following result gives more support to the heuristic argument.

Theorem 1.5. Let $r>0$ be a positive integer. Then Condition $C 1$ is satisfied for a generic matrix of rank $r$.

Referring to the above result by Rusek [26], we show that in fact a geometric equivalent formulation of $J C(\infty)$ is available, in terms of connectivity of some affine varieties.

Theorem 1.6. $J C(\infty)$ holds iff for all $n \in \mathbb{N}$ the affine variety $W_{n}^{\prime}:=\{(y, z, A) \in$ $W_{n}$ and $A$ is a Druzkowski matrix $\}$ is connected.

In particular, if $W_{n}$ is connected for every $n \in \mathbb{N}$ then $J C(\infty)$ holds.

In the remaining of this subsection, we state our main new reformulation of $J C(\infty)$ and some related results. These are again in line with the heuristic argument for the truth of the Jacobian conjecture mentioned above. Let $k$ be a positive integer in the range $\{1, \ldots, n\}$. We denote by $Z_{k}=(1, \ldots, 1,0, \ldots, 0)^{t}$ the vector whose first $k$ entries are 1 and whose last $n-k$ entries are 0 .

Conjecture NC. Let $A$ be an $n \times n$ Druzkowski matrix, $n \geq 3$. For each $k=$ $1, \ldots, n$, the equation $Z_{k}+A Z_{k}^{3}+A\left(Z_{k} *(A y)^{2}\right)=0$ has no solutions $y \in \mathbf{C}^{n}$.

The following result shows the significance of Conjecture NC.

Theorem 1.7. Conjecture $N C$ is equivalent to $J C(\infty)$.

We note that by the proof of Theorem 1.4, if $A$ satisfies the nilpotent condition $A^{2}=0$ (recall the further reduction by Druzkowski) then $A$ satisfies Conjecture NC. It will be shown in Lemma 4.4 that Conjecture NC is satisfied for small values $k=1$ and 2 .

In Section 2 we will illustrate the effectiveness (i.e. time saving) of using Conjecture $\mathrm{NC}$ and Conditions $\mathrm{C} 1$ and $\mathrm{C} 2$ on specific examples. In Section 5 we will explain theoretically this effectiveness and compare our approaches to several existing approaches. This explanation uses the following observation that a generic Druzkowski map automatically satisfies Conjecture NC. We note that a similar result holds if we employ Condition $\mathrm{C} 1$ instead of Condition C2.

Theorem 1.8. a) A generic Druzkowski map satisfies Conjecture NC.

b) More generally, if $V$ is a subvariety of the set of Druzkowski matrices and is invariant under the action of the group of invertible diagonal matrices, i.e. $D V D^{-3}=V$ for all invertible diagonal matrix $D$, then a generic element of $V$ satisfies Conjecture NC.

Among the many known reductions of the Jacobian conjecture, our Theorem 
1.3 and Conjecture $\mathrm{NC}$ are different and promising, both theoretically and computationally, for the following reasons.

- Hilbert's Nullstellensatz tells us how to show that a system of polynomial equations has no solutions. Recasting the Jacobian conjecture in terms of the solvability of such systems invites an effective computational approach, including numerical methods (see for example Section 5, Chapter 7 in [10]), to resolving the conjecture.

- For any Druzkowski matrix $A$, Theorem 1.8 shows that for a generic invertible diagonal matrix $D$, the Druzkowski matrix $D A D^{-3}$ satisfies Conjecture NC. Thus Conjecture NC holds for a generic Druzkowski matrix. As far as we know, Conjecture $\mathrm{NC}$ is the only equivalent formulation of $J C(\infty)$ that has been shown to hold with probability 1.

- Experimental results in Section 2.5 below shows that Theorem 1.3 provides a fast method to check whether a given Druzkowski matrix is an automorphism or not.

- The existing approaches to check the Jacobian conjecture for Druzkowski matrices usually employ either a nilpotent condition or induction on the rank of the matrix $A$. Our new reformulations provides new approaches, including using induction on $k$. This will be elaborated in more details in Section 5 .

The remaining of this paper is organised as follows. To illustrate the effectiveness of the methods, in Section 2, we present the experimental computations, including those on some Druzkowski matrices previously considered by other authors. In the next sections, we present theoretical results. In Section 3 we explain how Conditions $\mathrm{C} 1$ and $\mathrm{C} 2$ are derived, and why they imply the Jacobian Conjecture. We state there one generalisation of the Jacobian conjecture. In Section 4, we prove the above theorems. In Section 5, we give details of the approaches together with detailed strategies to employ them. In the same section we also explain theoretically the effectiveness (time saving) of our approaches in practice, compare them with other existing approaches, state some generalisations of the Jacobian conjecture together with both theoretical and experimental evidences to support them.

A longer version of this paper, with supplementary and additional facts and results and Mathematica codes, is available at the website [27].

\section{Results proven with the help of computer programs}

In this section we present the results we obtained with the help of computer programs. We have used two main resources: a Mathematica software run on a normal personal MacBook Air laptop and a MuPad software run on the BigMem cluster of the Tizzard super computer of eResearch SA. For the MuPad computations, the typical configuration is 1 node whose memory size is about $120 G B$ plus $100 G B$ virtual, and the working duration is about 4 to 5 days. We compute the Groebner basis of the corresponding polynomial systems, and (except Section 2.3) look to see whether $z_{1}, \ldots, z_{n}$ appear in the Groebner Basis. 
Remark. We will consider in this section Conditions $\mathrm{C} 1$ and $\mathrm{C} 2$ with the additional requirement $\operatorname{det}(A)=0$, which is satisfied by Druzkowski maps (the main focus of the Jacobian conjecture and this paper).

\subsection{The case $n=2$}

In this case, since $\operatorname{det}(A)=0$ we have that $A$ has rank 1 . Hence Condition $\mathrm{C} 1$ is satisfied.

\subsection{The case $n=3$}

Using Mathematica, we find that Condition $\mathrm{C} 1$ is true for $3 \times 3$ matrices $A$ with $\operatorname{det}(A)=0$.

\subsection{The case $n=4$, rank $=2$}

For the case $n=4$, the computation requires so long time and big memory that it does not terminate on a personal computer. Hence we have to use MuPad on the super computer. We found there is $(y, z, A)$ in $V_{4}$ (recall the notations $V_{n}$ and $W_{n}$ from the Introduction) such that $z=(1,1,1,0)$ and $A$ has rank 2. (This means that Condition $\mathrm{C} 1$ does not hold for general matrices, but we do not know whether it is true or not for Druzkowski matrices.) The time to compute the Groebner Basis was 9911 seconds, and the Groebner Basis has 179 elements. The Groebner Basis is too complicated (it takes more than 70 pages to print out) to extract any useful information at the moment. In particular, we cannot conclude whether there is such a counterexample with integer coefficients.

Using MuPad, we check that there is no $(y, z, A)$ in $W_{4}$ such that $z=(1,1,1,0)$ and $A$ has rank 2 . However, the check with $z=(1,1,1,1)$ could not terminate, hence the situation for Condition $\mathrm{C} 2$ is still unclear for us. Note that for a tuple $(y, z, A) \in W_{4}$, the matrix $A$ do not need to be Druzkowski. Hence, even if Condition $\mathrm{C} 2$ does not hold, it does not follow that Jacobian condition is not true. (Recall that Jacobian conjecture is equivalent to that Condition 2 holds for all Druzkowski matrices only, and not for more general matrices.)

\subsection{Random matrices with integer coefficients}

Before we proved the results in the Subsections 4.2 and 2.3, in a previous version we used Mathematica to investigate Condition C1 on randomly generated $4 \times 4$ matrices with integer coefficients in the interval $[-25,25]$. On all of those examples we found that Condition $\mathrm{C} 1$ is satisfied and the Groebner Basis has 6 elements.

From the results in Section 4.2 below, we know that for a generic matrix with integer coefficients then Condition $\mathrm{C} 1$ is satisfied. It is still open whether Condition $\mathrm{C} 1$ is satisfied by all matrices with integer coefficients. 
We have used MuPad to investigate higher dimensions and ranks. Below is a summary, the matrices here are randomly generated with integer coefficients lying between 0 and $10^{12}$ :

- $n=4$, rank $=3$ : The Groebner Basis has 8 elements, time to compute it is 16 seconds.

- $n=5$, rank $=3$ : The Groebner Basis has 9 elements, time to compute it is 358 seconds.

- $n=5$, rank $=4$ : The Groebner Basis has 15 elements, time to compute it is 15641 seconds.

For $n=10$, rank $=3$ or $n=7$, rank $=4$ the computations are usually terminated because of running out of time or memory, even when we restrict the entries to a smaller range.

\subsection{Some examples of Druzkowski matrices}

We present here the experiments with some examples of Druzkowski matrices previously considered by other authors. We recall that for a Druzkowski matrix $A$, then the matrix $\Delta\left[(A x)^{2}\right] . A$ is nilpotent. Then, its nilpotent index is the least positive integer $j$ such that $\left(\Delta\left[(A x)^{2}\right] \cdot A\right)^{j}=0$ for all $x \in X$. This notation has been used by Druzkowski in early papers on the subject of cubic linear maps. It has been shown first in [16] and then in [6], by different methods, that if the nilpotent index of $A$ is 3 then the corresponding Keller map satisfies the Jacobian conjecture.

Example 1. This example is taken from [20] (also page 140 in [19]), where Gorni and Zampieri developed their pairing between cubic homogeneous maps and cubic linear maps (see the citations for more details). In this example, $A$ is the following $15 \times 15$ matrix

$$
\left(\begin{array}{ccccccccccccccc}
0 & 0 & 0 & 0 & 0 & 0 & 0 & 0 & 0 & 0 & 0 & 0 & 0 & 0 & 0 \\
0 & 0 & 0 & 0 & 0 & 0 & 0 & 0 & 0 & 0 & 0 & 0 & 0 & 0 & 0 \\
0 & 0 & 0 & -2 & -1 & 1 & 1 & 1 & 0 & 0 & -1 & 0 & 0 & -1 & 0 \\
0 & 0 & -1 & 0 & -1 & 0 & 1 / 2 & 0 & 0 & 1 / 2 & 0 & -1 / 2 & -1 / 2 & 0 & 0 \\
0 & 0 & 1 & -2 & 0 & 0 & 0 & 1 & -1 & -1 & -1 & 0 & 0 & 0 & 1 \\
1 & 0 & 1 & -2 & 0 & 0 & 0 & 1 & -1 & -1 & -1 & 0 & 0 & 0 & 1 \\
0 & 1 & 1 & -2 & 0 & 0 & 0 & 1 & -1 & -1 & -1 & 0 & 0 & 0 & 1 \\
1 & 0 & -1 & 0 & -1 & 0 & 1 / 2 & 0 & 0 & 1 / 2 & 0 & -1 / 2 & -1 / 2 & 0 & 0 \\
1 & 0 & 0 & -2 & -1 & 1 & 1 & 1 & 0 & 0 & -1 & 0 & 0 & -1 & 0 \\
0 & 1 & 0 & -2 & -1 & 1 & 1 & 1 & 0 & 0 & -1 & 0 & 0 & -1 & 0 \\
1 & 0 & 1 & 0 & 1 & 0 & -1 / 2 & 0 & 0 & -1 / 2 & 0 & 1 / 2 & 1 / 2 & 0 & 0 \\
0 & 1 & -1 & 2 & 0 & 0 & 0 & -1 & 1 & 1 & 1 & 0 & 0 & 0 & -1 \\
0 & 1 & 0 & 2 & 1 & -1 & -1 & -1 & 0 & 0 & 1 & 0 & 0 & 1 & 0 \\
1 & 1 & 1 & -2 & 0 & 0 & 0 & 1 & -1 & -1 & -1 & 0 & 0 & 0 & 1 \\
1 & 1 & 0 & -2 & -1 & 1 & 1 & 1 & 0 & 0 & -1 & 0 & 0 & -1 & 0
\end{array}\right)
$$

This is a Druzkowski matrix and the corresponding Keller map satisfies the socalled globally analytically linearisable condition, whose conjugations are polyno- 
mial maps. We check that $A^{2}=0, A$ is of rank 5 and it has some non-zero $2 \times 2$ principal minor. Its nilpotent index is $>3$.

Using Mathematica, we check that this matrix $A$ satisfies Condition $\mathrm{C} 1$. The computation of the Groebner basis takes 9.62 seconds. We observe that when trying to check that the corresponding Druzkowski map is injective, an interesting phenomenon occurs. If we use the more obvious condition, that is $x+(A x)^{3}+$ $(A x) *(A y)^{2}=0$ implies $x=0$, then under the same setting (i.e. the same choice of monomial ordering as in the computation for Condition $\mathrm{C} 1$ ) it takes a very long time (610.90 seconds) to compute the Groebner basis. However, if we use the transformation in Lemma 3.1, that is $z+A \cdot\left(z^{3}+z *(A y)^{2}\right)=0$ implies $z=0$, it takes only 0.361 seconds to compute the Groebner basis.

Example 2. This example is taken from page 197 in [19]. Here $A$ is the following $17 \times 17$ matrix

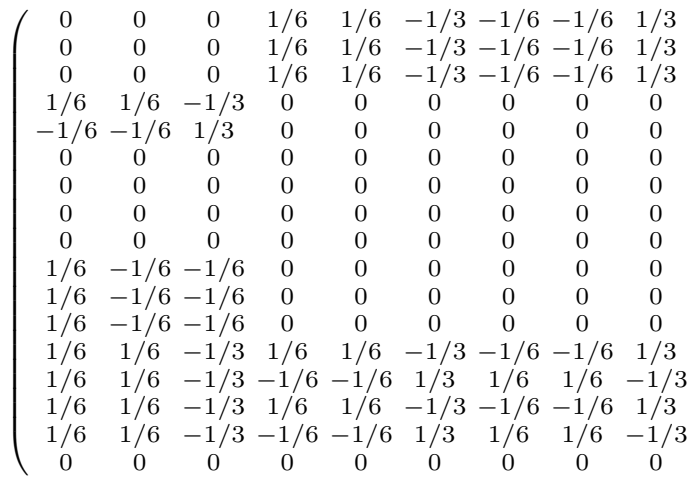

$\left.\begin{array}{cccccccc}0 & 0 & 0 & 0 & 0 & 0 & 0 & 1 \\ 0 & 0 & 0 & 0 & 0 & 0 & 0 & -1 \\ 0 & 0 & 0 & 0 & 0 & 0 & 0 & 0 \\ 0 & 0 & 0 & 0 & 0 & 0 & 0 & 1 \\ 0 & 0 & 0 & 0 & 0 & 0 & 0 & 1 \\ 0 & 0 & 0 & 0 & 0 & 0 & 0 & 1 \\ -1 / 6 & -1 / 6 & 1 / 3 & 1 / 12 & 1 / 12 & -1 / 12 & -1 / 12 & 1 \\ -1 / 6 & -1 / 6 & 1 / 3 & 1 / 12 & 1 / 12 & -1 / 12 & -1 / 12 & -1 \\ -1 / 6 & -1 / 6 & 1 / 3 & 1 / 12 & 1 / 12 & -1 / 12 & -1 / 12 & 0 \\ 0 & 0 & 0 & 0 & 0 & 0 & 0 & 1 \\ 0 & 0 & 0 & 0 & 0 & 0 & 0 & -1 \\ 0 & 0 & 0 & 0 & 0 & 0 & 0 & 0 \\ 0 & 0 & 0 & 0 & 0 & 0 & 0 & 1 \\ 0 & 0 & 0 & 0 & 0 & 0 & 0 & -1 \\ 0 & 0 & 0 & 0 & 0 & 0 & 0 & -1 \\ 0 & 0 & 0 & 0 & 0 & 0 & 0 & 1 \\ 0 & 0 & 0 & 0 & 0 & 0 & 0 & 0\end{array}\right)$

It is a Druzkowski matrix of rank 5 . While $A^{2} \neq 0, A^{3}=0$. The corresponding Keller map is a counterexample to the cubic-linear globally linearisation condition, mentioned in Example 1. The nilpotent index of $B$ is $>3$.

For this example, we do not know whether it satisfies Condition $\mathrm{C} 1$ or not (see, however, Section 5.1). Checking the Jacobian conjecture on this example is still very quick. Computing the Groebner Basis for the system $x+(A x)^{3}+(A x) *(A y)^{2}=0$ takes 0.577 seconds, and computing the Groebner Basis for the system $z+A .\left(z^{3}+\right.$ $\left.z *(A y)^{2}\right)=0$ takes 0.337 seconds.

One difference between this example and other examples considered in this subsection is that it has some non-zero principal $3 \times 3$ minors. We may speculate that because of this the computation of the Groebner Basis for Condition C1 takes a longer time. We also remark that Conjecture NC is satisfied on this example, and the time needed to compute it is quite fast. For example, with $k=17$ then the time needed to compute the Groebner Basis for the polynomial system in Conjecture NC is only 0.17979 seconds. This is what to be expected from Theorem 1.8.

Example 3. This example is taken from the paper [1], where the authors pro- 
posed an approach toward the Jacobian conjecture. Here $A$ is the $13 \times 13$ matrix

$$
\left(\begin{array}{ccccccccccccc}
0 & 0 & 0 & 1 / 6 & 1 / 6 & -1 / 3 & -1 / 6 & -1 / 6 & 1 / 3 & 0 & 0 & 0 & 1 \\
0 & 0 & 0 & 1 / 6 & 1 / 6 & -1 / 3 & -1 / 6 & -1 / 6 & 1 / 3 & 0 & 0 & 0 & -1 \\
0 & 0 & 0 & 1 / 6 & 1 / 6 & -1 / 3 & -1 / 6 & -1 / 6 & 1 / 3 & 0 & 0 & 0 & 0 \\
1 / 6 & 1 / 6 & -1 / 3 & 0 & 0 & 0 & 0 & 0 & 0 & 0 & 0 & 1 & 0 \\
1 / 6 & 1 / 6 & -1 / 3 & 0 & 0 & 0 & 0 & 0 & 0 & 0 & 0 & -1 & 0 \\
1 / 6 & 1 / 6 & -1 / 3 & 0 & 0 & 0 & 0 & 0 & 0 & 0 & 0 & 0 & 0 \\
0 & 0 & -1 / 3 & 0 & 0 & 0 & 0 & 0 & 0 & 1 / 6 & 1 / 6 & 0 & 1 \\
0 & 0 & -1 / 3 & 0 & 0 & 0 & 0 & 0 & 0 & 1 / 6 & 1 / 6 & 0 & -1 \\
0 & 0 & -1 / 3 & 0 & 0 & 0 & 0 & 0 & 0 & 1 / 6 & 1 / 6 & 0 & 0 \\
0 & 0 & 0 & 1 / 6 & 1 / 6 & -1 / 3 & -1 / 6 & -1 / 6 & 1 / 3 & 0 & 0 & 1 & 0 \\
0 & 0 & 0 & 1 / 6 & 1 / 6 & -1 / 3 & -1 / 6 & -1 / 6 & 1 / 3 & 0 & 0 & -1 & 0 \\
0 & 0 & 0 & 0 & 0 & 0 & 0 & 0 & 0 & 0 & 0 & 0 & 0 \\
0 & 0 & 0 & 0 & 0 & 0 & 0 & 0 & 0 & 0 & 0 & 0 & 0
\end{array}\right)
$$

This is a Druzkowski matrix of rank 5 and satisfies $A^{2}=0$. It has nilpotent index $>3$. For this example, the method in [1] consists of showing that certain 1170 Wronskians belong to a certain ring. The computation is quite involved and is contained in a big PDF file on the authors' website [2].

We have checked by Mathematica that this matrix satisfies Condition $\mathrm{C} 1$, and the computation of the Groebner Basis takes 0.137 seconds. Computing the Groebner Basis for the system $x+(A x)^{3}+(A x) *(A y)^{2}=0$ takes 0.535 seconds, and computing the Groebner Basis for the system $z+A \cdot\left(z^{3}+z *(A y)^{2}\right)=0$ takes 0.116 seconds.

We also did computations and obtained similar results with some other examples: a $4 \times 4$ Druzkowski matrix from [30] of nilpotent index 3 , and a $16 \times 16$ Druzkowski matrix from [20] of rank 4 and nilpotent index $>3$.

\section{Derivation of the Conditions}

In this section we explain the derivation of the Conditions $\mathrm{C} 1$ and $\mathrm{C} 2$ and show why they imply the Jacobian Conjecture. The plan is that we first prove Theorem 1.5. Then we state Conjecture (weaker version) and Conjecture (stronger version). By Lemma 3.1, we have that the condition on $W_{n}$ in Theorem 1.6 is equivalent to Conjecture (stronger version) for Druzkowski matrices. Hence Conjecture (stronger version) for Druzkowski matrices is equivalent to $J C(\infty)$. Since either $\mathrm{C} 1$ or $\mathrm{C} 2$ implies Conjecture (stronger version), it follows that either of them implies $J C(\infty)$.

There is a well-known result that a polynomial self-map of $\mathbb{C}^{n}$ is an automorphism if it is injective (Chapter 3 in [19]). In theory, we can check, for each given dimension $n$, whether all Druzkowski maps are injective by using a computer program (for example Mathematica) to find the Groebner basis for the ideal $\mathcal{I}$ defined by the equations $x+(A \cdot x)^{3}=y+(A . y)^{3}$ and

$$
\operatorname{det}\left(I d+\Delta\left[(A . z)^{2}\right] \cdot A\right)=1
$$


for all $z \in \mathbb{C}^{n}$, to see that $x-y$ belongs to this Groebner basis. However, in practice one faces the difficulty that the number of polynomials in the ideal defined by the system $\operatorname{det}\left(I d+\Delta\left[(A . z)^{2}\right] \cdot A\right)=1$ for every $z \in \mathbb{C}^{n}$ - with variables the $n^{2}$ entries of the matrix $A$, and hence consists of coefficients of $\operatorname{det}\left(I d+\Delta\left[(A . z)^{2}\right] \cdot A\right)=1$ regarded as a polynomial in $z$ - grows very fast with respect to the dimension $n$ : it is roughly the same as the number of monomials of degrees at most $n$ in $n$ variables. We note that an explicit procedure for writing down these equations was given in [21].

Remark. However, we note from the experiments (Section 2 above), that in practice it is quite effective to use Theorem 1.3 to check that any given Druzkowski map satisfies the Jacobian conjecture with the help of a computer program.

This paper grew out of the author's curiosity to see whether we can reduce the number of equations defining the ideal $\mathcal{I}$. (It is a classical result, [18] and references therein, that any algebraic subvariety of $\mathbb{C}^{N}$ is defined by $N$ equations, however it is quite challenging to find the equations for explicit examples.) It also originated from our trying to ponder on the following question:

Question. If a formal proof of the Jacobian conjecture is to be found for all Druzkowski maps of degree 3 in all dimensions $n$, how can we make use of the assumption that $J F$ is invertible?

To be more explicit about this Question, let us first make some simple algebraic reductions, which provide a proof of Theorem 1.3. Let $u, v \in \mathbb{C}^{n}$ be such that $F(u)=F(v)$, that is $u+(A \cdot u)^{3}=v+(A \cdot v)^{3}$. Then by subtracting and using that $A$ is a linear map, we find that

$$
(u-v)+(A \cdot u-A \cdot v) *\left((A \cdot u)^{2}+(A \cdot u) *(A \cdot v)+(A \cdot v)^{2}\right)=0 .
$$

If we define $x=u-v$ then we can write the above equation as

$$
x+(A \cdot x) *\left((A \cdot x)^{2}+3(A \cdot x) *(A \cdot v)+3(A \cdot v)^{2}\right)=0 .
$$

Now, by substituting

$$
y=\sqrt{3} v+\frac{\sqrt{3}}{2} x,
$$

and then replacing $x$ by $x / 2$, we see that the above equation is reduced to

$$
x+(A \cdot x) *\left((A \cdot x)^{2}+(A \cdot y)^{2}\right)=0 .
$$

Then, the fact that $F$ is injective is the same as that the equation $x+(A . x) *$ $\left((A \cdot x)^{2}+(A \cdot y)^{2}\right)=0$ has only the solution $x=0$.

We see that $y$ appears in the ideal $\mathcal{I}$ only through A.y. Hence it is natural to ask whether the Jacobian conjecture is in fact stronger, that is in Equation (3.2), we can replace $A y$ by $y$ (which of course must satisfy a condition compatible with Equation (3.1)) and still obtain the same conclusion? Hence, we state the following conjecture: 
Conjecture (weaker version). Let $A$ be a Druzkowski $n \times n$ matrix. Assume that $y \in \mathbb{C}^{n}$ satisfy

$$
\operatorname{det}\left(I d+\Delta\left[(A \cdot x+t y)^{2}\right] \cdot A\right)=1
$$

for all $t \in \mathbb{C}$ and all $x \in \mathbb{C}^{n}$. Then, if $x+(A x) *\left((A x)^{2}+y^{2}\right)=0$, we must have $x=0$.

Remark. If $A$ is an $n \times n$ matrix for which $\operatorname{det}\left(I d+\Delta\left[(A \cdot x+t y)^{2}\right] \cdot A\right)=1$ for all $t \in \mathbb{C}$ and $x \in \mathbb{C}^{n}$ then $A$ must be a Druzkowski map as we can readily see by putting $t=0$ in the equality. Hence the above Conjecture, while a bit stronger than the Jacobian conjecture, is only for Druzkowski matrices.

We may push this investigation further, by asking that in showing that $x+$ $(A . x) *\left((A \cdot x)^{2}+y^{2}\right)=0$ has only the solution $x=0$, do we need the assumption (3.1) somehow on the plane generated by $A . x$ and $y$ only? This leads us to state a stronger conjecture.

Conjecture (stronger version). Let $A$ be a Druzkowski $n \times n$ matrix. Assume that $x, y \in \mathbb{C}^{n}$ satisfy

$$
\operatorname{det}\left(I d+\Delta\left[(s A \cdot x+t y)^{2}\right] \cdot A\right)=1
$$

for all $s, t \in \mathbb{C}$. Then, if moreover $x+(A x) *\left((A x)^{2}+y^{2}\right)=0$, we must have $x=0$.

These two conjectures can be seen to be more general than the original Jacobian conjecture. If we ask for not only Druzkowski matrices but general $n \times n$ matrices and use the following Lemma, we arrive at Condition C1, by substituting A.x in the statement of Conjecture (stronger version) by a new variable $z$.

Lemma 3.1. The following two statements are equivalent:

1) There is a non-zero solution $x$ to $x+(A \cdot x)^{3}+(A . x) * y^{2}=0$, and

2) There is a non-zero solution $z$ to $z+A \cdot\left(z^{3}+z * y^{2}\right)=0$.

Proof. $(\Rightarrow)$ If $x$ is a non-zero solution to $x+(A \cdot x)^{3}+(A \cdot x) * y^{2}=0$ then $z=A \cdot x$ is non-zero. Moreover, we have

$$
\begin{aligned}
0 & =A\left(x+(A . x)^{3}+(A . x) * y^{2}\right)=A\left(x+z^{3}+z * y^{2}\right) \\
& =A(x)+A\left(z^{3}+z * y^{2}\right)=z+A\left(z^{3}+z * y^{2}\right) .
\end{aligned}
$$

$(\Leftarrow)$ If $z$ is a non-zero solution to $z+A\left(z^{3}+z * y^{2}\right)=0$, by defining $x=$ $-\left(z^{3}+z^{*} y^{2}\right)$ we see that $A x=z$. In particular, $x$ is also non-zero. Moreover,

$$
0=x+z^{3}+z * y^{2}=x+(A \cdot x)^{3}+(A \cdot x) * y^{2} .
$$

Experimental evidences in Section 2 support these Conjectures. In Section 5.1 we give theoretical reasons to support these Conjectures. 


\section{General properties}

In the first subsection of this section, we prove Theorems 1.4, 1.6 and 1.8. The proofs of Theorems 1.7 and 1.5 are given in the second subsection, where we also prove some other results. In the third subsection, we give a simple and direct proof of the fact that symmetric Druzkowski maps satisfy the Jacobian conjecture.

\subsection{Proofs of Theorems 1.4, 1.6 and 1.8}

Proof of Theorem 1.4. Assume that $\Delta\left[(A x)^{2}\right] \cdot A=B . \Delta\left[(A x)^{2}\right] J$, where $J$ is a diagonal matrix, and both $B$ and $J$ are independent of $x$. Then, for all $x, y \in \mathbb{C}^{n}$, since diagonal matrices commute we have:

$$
\text { A. } \begin{aligned}
\Delta\left[(A x)^{2}\right] \cdot A \cdot \Delta\left[(A y)^{2}\right] & =A \cdot B \cdot \Delta\left[(A x)^{2}\right] \cdot J \cdot \Delta\left[(A y)^{2}\right] \\
& =A \cdot B \cdot \Delta\left[(A y)^{2}\right] \cdot J \Delta\left[(A x)^{2}\right] \\
& =A \cdot \Delta\left[(A y)^{2}\right] \cdot A \cdot \Delta\left[(A x)^{2}\right] .
\end{aligned}
$$

In other words, $A . \Delta\left[(A x)^{2}\right]$ and $A . \Delta\left[(A y)^{2}\right]$ commute. Moreover, if $(y, z, A) \in W_{n}$, then $A . \Delta\left[y^{2}\right]$ is nilpotent. We can proceed as in Section 4.3 below.

Proof of Theorem 1.6. If $J C(n)$ holds then $W_{n}^{\prime}=\left\{(y, 0, A): y \in \mathbb{C}^{n}\right.$ and $A$ is a Druzkowski matrix $\}$, and hence is connected since the set of Druzkowski matrices is connected. (If $A$ is a Druzkowski matrix then $t A$ is also a Druzkowski matrix for any $t \in \mathbb{C}$. In particular, there is a path connecting $A$ and 0.)

For the proof of the reverse implication, we first show that if $V$ is an irreducible component of $V_{n}$ or $W_{n}$ such that $V \cap\{z=0\} \neq \emptyset$ then $V \subset\{z=0\}$. Assume that there is $(y, 0, A) \in V$ and a sequence $\left(y^{(j)}, z^{(j)}, A^{(j)}\right) \in V$ such that

$$
\begin{aligned}
z^{(j)} & \neq 0, \forall j, \\
\left(y^{(j)}, z^{(j)}, A^{(j)}\right) & \rightarrow(y, 0, A) .
\end{aligned}
$$

We will show a contradiction.

We define, as in the proof of Lemma 3.1,

$$
x^{(j)}=-z^{(j)} * z^{(j)} * z^{(j)}-z^{(j)} * y^{(j)} * y^{(j)} .
$$

Then $x^{(j)} \neq 0$ for all $j$ and $x^{(j)} \rightarrow 0$. Moreover, $x^{(j)}+\left(A^{(j)} x^{(j)}\right)^{3}+\left(A^{(j)} x^{(j)}\right) \cdot y^{(j)} *$ $y^{(j)}=0$ for all $j$. We can rewrite this equation as

$$
\left(I d+A^{(j)} \cdot \Delta\left[\left(A^{(j)} \cdot y^{(j)}\right)^{2}\right]\right) \cdot x^{(j)}=-\left(A^{(j)} \cdot x^{(j)}\right)^{3} .
$$

Since $\left(y^{(j)}, z^{(j)}, A^{(j)}\right) \in V_{n}$, it follows that $\operatorname{det}\left(I d+A^{(j)} \cdot \Delta\left[\left(A^{(j)} \cdot y^{(j)}\right)^{2}\right]\right)=1$ for all $j$ (see the proof of Lemma 3.1 for more details). The fact that $\left(y^{(j)}, z^{(j)}, A^{(j)}\right.$ ) converges to $(y, 0, A)$ implies that the inverse matrices $\left(I d+A^{(j)} \cdot \Delta\left[\left(A^{(j)} \cdot y^{(j)}\right)^{2}\right]\right)^{-1}$ are bounded. From

$$
x^{(j)}=-\left(I d+A^{(j)} \cdot \Delta\left[\left(A^{(j)} y^{(j)}\right)^{2}\right]\right)^{-1} \cdot\left(A^{(j)} x^{(j)}\right)^{3},
$$


it follows that $\left\|x^{(j)}\right\| \leq C\left\|x^{(j)}\right\|^{3}$ for some positive constant independent of $j$. The assumption that $x^{(j)} \rightarrow 0$ then implies that $x^{(j)}=0$ for large $j$, as wanted.

We now finish the proof of Theorem 1.6. If $W_{n}^{\prime}$ is connected, then the above paragraph shows that $W_{n}^{\prime} \subset\{z=0\}$ and hence $J C(n)$ holds. Finally, if $W_{n}$ is connected, then $W_{n} \subset\{z=0\}$, and hence so is $W_{n}^{\prime}$.

Proof of Theorem 1.8. We give only the proof of a), since the proof of b) is identical.

We will use the arguments and notations of the next subsection. Let $A$ be a Druzkowski matrix. Then the set consisting of all matrices of the form $D A D^{-3}$, where $D$ runs over all invertible diagonal matrices, belongs to the same irreducible component of all Druzkowski matrices. (In fact, let $f$ be the map from the set of invertible diagonal matrices to the set of Druzkowski matrices defined by $D \mapsto$ $D A D^{-3}$. This is a regular morphism between algebraic varieties. Since the set of invertible diagonal matrices is irreducible, it follows that there is an irreducible component $W$ of the set of Druzkowski matrices for which $f^{-1}(W)$ is the whole of invertible diagonal matrices.) We need to show only that at least one among these matrices satisfy Conjecture NC. Assume otherwise. Then, by Lemma 4.4 below, in particular $A$ does not satisfy Conjecture NC with respect to some $k \in\{3, \ldots, n\}$.

Let $A_{1,1}$ be the $k \times k$ submatrix of $A$ as in the next subsection. Then the arguments in the next section shows that $A_{1,1}$ is nilpotent, in particular is of rank $<k$. We will show that for a generic choice of the invertible diagonal matrix $D$, then $(1, \ldots, 1)^{t}$ does not belong to the image of $D_{1} A_{1,1} D_{1}^{-3}$ where $D_{1}$ is the $k \times k$ submatrix of $D$ as in the next subsection. Therefore, for such a choice of $D$, there is no $y$ for which $\left(y, z=Z_{k}, D A D^{-3}\right) \in V_{n}$, as wanted. Assume that this is not the case, we will deduce a contradiction.

In fact, assume that for all invertible diagonal matrix $D$ then $(1, \ldots, 1)^{t}$ belongs to the image of $D_{1} A_{1,1} D_{1}^{-3}$. Then we see that $D_{1}^{-1}(1, \ldots, 1)^{t}$ belongs to the image of $A_{1,1} D_{1}^{-3}$ and hence to the image of $A_{1,1}$ for all such $D$. But the set of all such vectors $D_{1}^{-1} \cdot(1, \ldots, 1)^{t}$ is exactly the set $\left\{\left(x_{1}, \ldots, x_{k}\right) \in \mathbb{C}^{k}: x_{1} \ldots x_{k} \neq 0\right\}$. Since $A_{1,1}$ is nilpotent, it cannot contain all of this set. This gives a contradiction as desired.

Then the intersection of all these generic sets, when $k$ runs over all the set $\{3, \ldots, n\}$, is still a generic set. All matrices in this intersection set satisfies Conjecture NC for all $k \in\{3, \ldots, n\}$.

Finally, using the properties of the projections of affine algebraic varieties (in particular, the Closure Theorem in Section 6, Chapter 5 in [11]), we conclude that there is a proper subvariety (and moreover does not contain any irreducible component) of the set of all Druzkowski matrices outside which Conjecture NC holds $\square$ 


\subsection{More matrices satisfying Condition $C 1$}

Note that $V_{n}$ (and $W_{n}$ ) are invariant under the group action by invertible diagonal matrices (see the paragraph after the statement of Theorem 1.4): $(y, z, A) \mapsto$ $\left(D y, D z, D A D^{-3}\right)$. Hence, we can reduce the study of $V_{n}$ (and $W_{n}$ ) to a simpler case as follows. Let $(y, z, A)$ be in $V_{n}$. Let us define $w=\left(w_{1}, \ldots, w_{n}\right)^{t}$ where $w_{i}$ is an arbitrary nonzero complex number if $z_{i}=0$, and $w_{i}=1 / z_{i}$ otherwise. Then the diagonal matrix $D=\Delta[w]$ is invertible, $\left(y^{\prime}, z^{\prime}, A^{\prime}\right)=\left(D y, D z, D A D^{-3}\right)$ is also in $V_{n}$ and $z^{\prime}$ has the special form concerned in Conjecture NC: coordinates of $z^{\prime}$ are either 0 or 1 . In particular, $\left(z^{\prime}\right)^{2}=z^{\prime}$.

Fix $r>0$ a positive integer. Let us choose $(y, z, A)$ an element in $V_{n}$ such that $z^{2}=z$ and $A$ is of rank $r$. After a permutation, we can assume that $z=$ $(1, \ldots, 1,0, \ldots, 0)^{t}$ has the first $k$ entries to be 1 and the last $n-k$ entries to be 0 . Moreover, if we choose $w$ in the above appropriately, we can assume that $y$ satisfies additionally the constraints $y_{k+1}^{2}=y_{k}, \ldots, y_{n}^{2}=y_{n}$. This gives the proof of Theorem 1.7. Indeed, by Theorem 1.3 and Lemma 3.1, $J C(\infty)$ does not hold then there is a Druzkowski matrix $A$ and a pair $y, z$ such that $z+A z^{3}+A\left(z *(A y)^{2}\right)=0$ and $z \neq 0$. Then the above arguments show that we can choose $z$ to be $Z_{k}$, where $k$ is the number of non-zero coordinates of $z$. Hence, Conjecture NC also does not hold. On the other hand, we note that Conjecture $\mathrm{NC}$ is indeed a special case of $J C(\infty)$. Hence $J C(\infty)$ and Conjecture $\mathrm{NC}$ are equivalent.

We write $A$ in the block form

$$
\left(\begin{array}{ll}
A_{1,1} & A_{1,2} \\
A_{2,1} & A_{2,2}
\end{array}\right)
$$

where $A_{1,1}$ is of the size $k \times k$. The set $\mathcal{E}_{r}$ of $n \times n$ matrices for which all minors up to dimension $r$ are non-zero is dense in the set of all matrices of rank $r$, hence we can consider only these matrices. We now deduce two simple conditions which $A$ must satisfy if $(y, z, A) \in V_{n}$.

Lemma 4.1. Assume that $A$ has the above block form, and $z=(1, \ldots, 1,0, \ldots, 0)^{t}$ as above. Then:

1) $A_{1,1}$ is a nilpotent $k \times k$ matrix and has rank exactly $r$.

2) $(1, \ldots, 1)^{t}$ ( $k$ entries) is in the image of $A_{1,1}$.

Proof. 1) In the condition $\operatorname{det}\left(I d+\Delta\left[(s z+t y)^{2}\right] . A\right)=1$ for all $s, t \in \mathbb{C}$, if we choose $t=0$ we see that $\operatorname{det}\left(I d+s^{2} \Delta\left[z^{2}\right] . A\right)=1$ for all $s$, and hence $\Delta\left[z^{2}\right] . A$ is nilpotent. Since $z=(1, \ldots, 1,0, \ldots, 0)^{t}$, we deduce that $A_{1,1}$ is a nilpotent $k \times k$ matrix. Since $A \in \mathcal{E}_{r}$, it follows that $k \geq r$, and hence $A_{1,1}$ has rank exactly $r$.

2) Since $z+A \cdot\left(z^{3}+z * y^{2}\right)=0$, we deduce in particular that $z$ is in the image of $A$. Since $z=(1, \ldots, 1,0 \ldots, 0)^{t}$, it follows moreover that $(1, \ldots 1)^{t}$ is in the image of $A_{1,1}$.

If $k \geq 2$ then the set of all $k \times k$ matrices $A_{1,1}$ satisfying the above two conditions 
is a very small set (more specifically, of high codimension) in the set of all $k \times k$ matrices of rank $r$. We formally state and prove this statement.

Lemma 4.2. The set of $k \times k$ matrices $A_{1,1}$ of rank exactly $r$ satisfying conclusions 1) and 2) in Lemma 4.1 is of codimension at least $k$ in the set of all $k \times k$ matrices of rank $r$.

\section{Proof.}

Any nilpotent $k \times k$ matrix $A_{1,1}$ of rank exactly $r$ can be written as $A_{1,1}=$ $D^{-1} J D$, where $D$ is an invertible matrix and $J$ is a nilpotent matrix of Jordan form of rank exactly $r$. Since there are only a finite number of matrices of Jordan form, it is sufficient to prove the claim for nilpotent matrices $A_{1,1}$ with a fixed such Jordan form $J$. To this end, for a fixed non-zero vector $v$, we will show that the set $\Gamma_{v}$ of such nilpotent matrices whose image contains $v$ has dimension at most $2 k r-r^{2}-k$. Note that $2 k r-r^{2}$ is the dimension of the set of $k \times k$ matrices of rank exactly $r$.

It can be checked easily that $v$ is in the image of $A_{1,1}$ iff $D v$ is in the image of $J$. The set of all matrices $D$ with this property consists of invertible elements in a vector space $\mathcal{S}$ of square matrices of dimension exactly $k^{2}-(k-r)$. We note that two such matrices $D_{1}^{-1} J D_{1}$ and $D_{2}^{-1} J D_{2}$ are the same iff $D=D_{1} D_{2}^{-1}$ is in the group $G$ of invertible matrices commuting with $J$. Thus the dimension of $\Gamma_{v}$ is exactly

$$
\operatorname{dim}(\mathcal{S})-\operatorname{dim}(G)=k^{2}-(k-r)-\operatorname{dim}(G) .
$$

Hence we need to show that

$$
\operatorname{dim}(G) \geq k^{2}-(k-r)-\left(2 k r-r^{2}-k\right)=k^{2}-2 k r+r^{2}+r=(k-r)^{2}+r .
$$

The dimension of $G$ can be determined easily from the sizes of the blocks of the Jordan matrix $J$, from the classical results in the theory of matrix group (see e.g. Section 1.2 in [23]). We recall here how to compute it. Let $W_{1}, \ldots, W_{l}$ be the blocks of the Jordan matrix $J$ of sizes $\mu_{1}, \ldots, \mu_{l}$. Then $\mu=\left(\mu_{1}, \ldots, \mu_{l}\right)$ is a partition of $k$, i.e. $\mu_{1}+\ldots+\mu_{l}=k$. The dual partition of $\mu$ is given by $\nu=\left(\nu_{1}, \ldots, \nu_{k}\right)$, where $\nu_{i}=$ the number of $j$ 's for which $\mu_{j} \geq i$. (Here $\nu$ may not be a partition in the usual sense since some of its elements may be 0 .) Then

$$
\operatorname{dim}(G)=\nu_{1}^{2}+\ldots+\nu_{k}^{2} .
$$

Since $J$ has rank exactly $r$, it follows that

$$
\left(\mu_{1}-1\right)+\ldots+\left(\mu_{l}-1\right)=r
$$

from this we get $r+l=k$, or $l=k-r$. Thus $\nu_{1}=l=k-r$. Therefore, $\nu_{2}+\ldots+\nu_{k}=$ $r$. From this we obtain

$$
\operatorname{dim}(G) \geq \nu_{1}^{2}+\left(\nu_{2}+\ldots+\nu_{l}\right)=(k-r)^{2}+r,
$$

as wanted. 
In the above, we showed that the set $\Phi_{k}=\{A:$ there exist $y, z$ so that $(y, z, A) \in V_{n}$ and $\left.z=(1, \ldots, 1,0, \ldots, 0)^{t}\right\} \subset \Lambda_{k}, k \geq 2$, where $\Lambda_{k}$ has codimension at least $k$ in the set of all matrices. From the beginning of this subsection, we also saw that if $\widehat{\Phi_{k}}=\left\{(y, z, A) \in V_{n}\right.$ where $z$ has exactly $k$ non-zero coordinates $\}$, then for every $(y, z, A) \in \widehat{\Phi_{k}}$ - after a permutation of coordinates - there is an invertible matrix $D$ so that $D A D^{-3} \in \Phi_{k}$. We now show that $\widehat{\Phi_{k}}$ has codimension at least 1 in the set of all matrices.

Lemma 4.3. $\widehat{\Phi_{k}}$ has codimension at least 1 in the set of all matrices.

Proof. Define $\widehat{\Lambda_{k}}=\left\{D A D^{-3}: A \in \Lambda_{k}, D:\right.$ invertible diagonal matrix $\}$. Then $\widehat{\Phi_{k}} \subset \widehat{\Lambda_{k}}$, and hence it is enough to show that $\widehat{\Lambda_{k}}$ has codimension at least 1 in the set of all matrices.

Let $A \in \Lambda_{k}$ with the block form as above, and $D$ be an invertible diagonal $n \times n$ matrix. If $D$ has the block form

$$
\left(\begin{array}{cc}
D_{1} & 0 \\
0 & D_{2}
\end{array}\right)
$$

where $D_{1}$ is of size $k \times k$, then $D A D^{-3}$ has the block form

$$
\left(\begin{array}{cc}
D_{1} A_{1,1} D_{1}^{-3} & D_{1} A_{1,2} D_{2}^{-3} \\
D_{2} A_{2,1} D_{1}^{-3} & D_{2} A_{2,2} D_{2}^{-3}
\end{array}\right)
$$

Since $\Lambda_{k}$ is defined by $k$ homogeneous equations and the set of all diagonal matrices $D_{1}$ is of dimension $k$, the total dimension of the orbit of $\Lambda_{k}$ is only $=$ $\operatorname{dim}(\Lambda)+k-1$ (and not $\operatorname{dim}(\Lambda)+k$ ). Hence, by Lemma $4.2, \widehat{\Lambda_{k}}$ is of codimension at least 1 in the set of all $k \times k$ matrices.

Now we prove Theorem 1.5. We need to show that the set of matrices $A$ so that there is $y, z$ such that $(y, z, A) \in V_{n}$ and $z \neq 0$, is of codimension at least 1 in the set of all matrices. Indeed, choose $(y, z, A) \in V_{n}$, where $z$ has exactly $k \geq 1$ non-zero coordinates. By Lemma 4.3 , if $k \geq 2$, then we are done. The next Lemma deals with the remaining case $k=1$.

Lemma 4.4. Let $k=1$ or 2 . There is no $(y, z, A)$ in $V_{n}$ with $z=(1, \ldots, 1,0, \ldots, 0)$ where the first $k$ entries are 1 and the last $n-k$ entries are 0.

Proof. We first consider the case $k=1$. Assume that there is $(y, z, A)$ in $V_{n}$ where $z=(1,0, \ldots, 0)$. Then, there is $s \in \mathbb{C}$ such that $(s z+y)^{2}=z^{2}+y^{2}$. From this we have

$$
\begin{aligned}
0 & =z+A \cdot\left(z^{3}+z * y^{2}\right)=\left(I d+A \cdot \Delta\left[z^{2}+y^{2}\right]\right) \cdot z \\
& =\left(I d+A \cdot \Delta\left[(s z+y)^{2}\right]\right) \cdot z
\end{aligned}
$$

which will imply $z=0$ provided that $I d+A \cdot \Delta\left[(s z+y)^{2}\right]$ is invertible. To this end, it suffices to show that $\operatorname{det}\left(I d+A \cdot \Delta\left[(s z+t y)^{2}\right]\right)=1$ for all $s, t \in \mathbb{C}$. In fact, 
since $(y, z, A)$ is in $V_{n}$, we have that $\operatorname{det}(I d+D(s, t) \cdot A)=0$ for all $s, t \in \mathbb{C}$, where $D(s, t)=\Delta\left[(s z+t y)^{2}\right]$. The latter is the same as $D(s, t) . A$ is nilpotent, that is $(D(s, t) \cdot A)^{n}=0$. Then,

$$
(A \cdot D(s, t))^{n+1}=A \cdot(D(s, t) \cdot A)^{n} \cdot D(s, t)=0,
$$

which implies that $A . D(s, t)$ is also nilpotent for every $s, t$. This then implies that $\operatorname{det}(I d+A \cdot D(s, t))=1$ for all $s, t$ as wanted.

It remains to consider the case $k=2$. In this case, we write $A$ in the block form as

$$
\left(\begin{array}{ll}
A_{1,1} & A_{1,2} \\
A_{2,1} & A_{2,2}
\end{array}\right)
$$

where

$$
A_{1,1}=\left(\begin{array}{ll}
a & b \\
c & d
\end{array}\right)
$$

is a $2 \times 2$ matrix. We have as before $A_{1,1}^{2}=0$ and

$$
(1,1)^{t}+A_{1,1} \cdot\left(1+y_{1}^{2}+y_{2}^{2}\right)^{t}=0 .
$$

Multiplying the above system with $A_{1,1}$, using that $A_{1,1}^{2}=0$, we find that

$$
c+d=b-d=a+d=-1+d\left(y_{1}^{2}-y_{2}^{2}\right)=0 .
$$

Now consider again the condition $\operatorname{det}\left(I d+\Delta\left[(s z+t y)^{2}\right]\right)=0$ for all $s, t \in \mathbb{C}$. Expanding the left hand side as a polynomial in variables $s, t$, we have that its homogeneous part of degree 2 , which is

$$
a_{1,1}\left(s+t y_{1}\right)^{2}+a_{2,2}\left(s+t y_{2}\right)^{2}+a_{3,3}\left(t y_{3}\right)^{2}+\ldots+a_{n, n}\left(t y_{n}\right)^{2},
$$

must be 0 . In particular, consider the coefficient of the term st, we find that $a_{1,1} y_{1}+$ $a_{2,2} y_{2}=-d\left(y_{1}-y_{2}\right)$ must be 0 . This contradicts the condition $-1+d\left(y_{1}^{2}-y_{2}^{2}\right)=0$ we found in the above.

The conclusion of Lemma 4.4 does not hold for the case $k=3$ and bigger, see Section 2. For Druzkowski maps, see however Sections 3 and 5.1.

The set of matrices which satisfies Condition $\mathrm{C} 1$ is big, and is dense in the set of matrices of a given rank $r$. Here is an explicit calculation illustrating the above arguments. Here we illustrate the situation when $n=2$. In this case, by the same argument as that of Lemma 4.4, the set of matrices $A$ which do not satisfy Condition $C_{1}$ is the set of all $2 \times 2$ matrices $A$ of the form $A=D A_{0} D^{-3}$, where $D$ is an invertible diagonal matrix and $A_{0}$ is the matrix

$$
\left(\begin{array}{ll}
1 & -1 \\
1-1
\end{array}\right)
$$

Hence this set is only of dimension 2 , and since the set of $2 \times 2$ matrices of rank at most 1 has dimension 3, we see that almost all matrices satisfy Condition $C 1$. 


\subsection{Application: The case of symmetric Druzkowski maps}

A Druzkowski map $F_{A}(x)=x+(A x)^{3}$ is called symmetric if its Jacobian is a symmetric matrix. This is the same as that $\Delta\left[(A x)^{2}\right] . A$ is a symmetric matrix for all $x \in \mathbb{C}^{n}$. In [5] and [13], it was shown that symmetric Druzkowski maps satisfy the Jacobian conjecture. The proofs in these two papers are quite similar, and proceed by induction on the dimension $n$. In this subsection, we give a simpler and more direct proof of this fact.

Proof. Assume that $F_{A}(x)$ is a symmetric Druzkowski map. To show that $F_{A}$ satisfies the Jacobian conjecture, it suffices to show that if $z+A\left(z^{3}\right)+A .\left((A y)^{2} * z\right)=$ 0 then $z=0$.

First, we observe that

$$
A \cdot\left[\Delta(A x)^{2}\right] \cdot A \cdot\left[\Delta(A y)^{2}\right]=A \cdot\left[\Delta(A y)^{2}\right] \cdot A \cdot\left[\Delta(A x)^{2}\right]
$$

for all $x, y \in \mathbb{C}^{n}$. In fact, because $F_{A}$ is symmetric and $\Delta\left[(A x)^{2}\right]$ is a diagonal matrix, we find that

$$
\Delta\left[(A x)^{2}\right] \cdot A=\left(\Delta\left[(A x)^{2}\right] \cdot A\right)^{t}=A^{t} \cdot \Delta\left[(A x)^{2}\right] .
$$

Here $(.)^{t}$ means the transpose of a matrix. Applying this and using that diagonal matrices commute we have

$$
\begin{aligned}
A \cdot\left[\Delta(A x)^{2}\right] \cdot A \cdot\left[\Delta(A y)^{2}\right] & =A \cdot A^{t} \cdot\left[\Delta(A x)^{2}\right] \cdot\left[\Delta(A y)^{2}\right] \\
& =A \cdot A^{t} \cdot\left[\Delta(A y)^{2}\right] \cdot\left[\Delta(A x)^{2}\right] \\
& =A \cdot\left[\Delta(A y)^{2}\right] \cdot A \cdot\left[\Delta(A x)^{2}\right] .
\end{aligned}
$$

Using this observation, now we finish the proof, i.e. showing that if $z+A\left(z^{3}\right)+$ $A .\left((A y)^{2} \cdot z\right)=0$ then $z=0$. Note that $z$ in the image of $A$, hence we can write $z=A x$ for some $x$. Since $F_{A}$ is a Druzkowski map, we have $\left(A . \Delta\left[z^{2}\right]\right)^{n}=$ $\left(A . \Delta\left[(A x)^{2}\right]\right)^{n}=0$. The equation for $z$ implies that

$$
z=-\left(I d+A \cdot \Delta\left[z^{2}\right]\right)^{-1} \cdot\left(A \cdot \Delta\left[(A y)^{2}\right]\right) \cdot z .
$$

Hence we are done if it can be shown that $\left(I d+A \cdot \Delta\left[z^{2}\right]\right)^{-1} \cdot\left(A \cdot \Delta\left[(A y)^{2}\right]\right)$ is a nilpotent matrix. The latter fact is equivalent to that $\left(A . \Delta\left[(A y)^{2}\right]\right) \cdot\left(I d+A . \Delta\left[z^{2}\right]\right)$, and hence $\left(I d+A \cdot \Delta\left[z^{2}\right]\right) \cdot\left(A \cdot \Delta\left[(A y)^{2}\right]\right)$, is nilpotent. Using the fact proven above that $A . \Delta\left[z^{2}\right]$ and $A . \Delta\left[(A y)^{2}\right]$ commute, we find that

$$
\left[\left(I d+A \cdot \Delta\left[z^{2}\right]\right) \cdot\left(A \cdot \Delta\left[(A y)^{2}\right]\right)\right]^{n}=\left(I d+A \cdot \Delta\left[z^{2}\right]\right)^{n} \cdot\left(A \cdot \Delta\left[(A y)^{2}\right]\right)^{n}=0 .
$$

The last equality follows since $A$ is a Druzkowski matrix.

\section{Remarks.}

1) Besides showing that symmetric Druzkowski maps satisfy the Jacobian conjecture, [5] and [13] also classified all such maps. While our proof does not do so, it also proves the more general result of Theorem 1.4. Indeed, the first part of the above proof is only to establish that $A . \Delta\left[x^{2}\right]$ and $A . \Delta\left[y^{2}\right]$ commute for all $x, y$, and 
then the last part of the above proof uses only this commutativity and the fact that A. $\Delta\left[(A y)^{2}\right]$ is nilpotent. In the proof indicated in Subsection 4.1, we also established these needed conditions, and hence the remaining of the proof of Theorem 1.4 can proceed as here.

2) Meisters [24] defines a matrix $A$ to be beautiful if $\Delta[A x] \cdot \Delta[A y] . A$ is nilpotent for all $x, y \in \mathbb{C}^{n}$, which is similar to the condition that $(I d+$ $\left.\Delta\left[(A x)^{2}\right] . A\right)^{-1} \cdot\left(\Delta\left[(A y)^{2}\right] . A\right)$ is nilpotent which we used in the above proof. We note that both of these conditions are closed under taking limit in the usual topology on the entries of the square matrices, and are not satisfied by Example 1 in Section 2.5 above.

\section{Some approaches}

We propose in this section several approaches towards resolving the Jacobian conjecture and detailed strategies for implementing them. We start with some comments on the effectiveness of the approaches proposed and the comparisons to several existing approaches of other authors.

\subsection{The effectiveness of our reformulations and comparisons to existing approaches}

In this subsection we explain theoretically why the Conditions $\mathrm{C} 1$ and $\mathrm{C} 2$, and Theorems 1.7 and 1.8 are quite effective in practice. We also compare our approach to several existing approaches.

In many of the existing approaches, a common theme is to construct the inverse of a given Keller map. Usually, this is achieved by writing out a formula for the inverse and giving a bound for the degree of the inverse. The bound is then translated into a corresponding system of polynomials. For example, the derivation criterion Proposition 1.2 is of this nature, and the bound there is $d^{n-1}$ which grows exponentially. For a Druzkowski map in dimension $n$ this bound is $3^{n-1}$ which is quite big even if $n$ is small. There are several approaches (for example [16] and [1]) which reduce the complexity. However, as illustrated in [1] (see Example 3 in Section 2.5 above), in practice the computations following this "finding inverse" approach are still quite large.

Our approach is to check instead only the injectivity and does not try to construct the inverse map. For a general map, checking whether it is injective may be not any easier than finding its inverse. However, this turns out to be very suitable for Druzkowski maps, because of special properties of Linear Algebra. Here, given an $n \times n$ matrix $A$, we only need to check that a system of $n$ equations of degree 3 in $2 n$ variables $y_{1}, \ldots, y_{n}$ and $z_{1}, \ldots, z_{n}$ has a very simple solution set, meaning $\left\{z_{1}=\ldots=z_{n}=0\right\}$. Moreover, the application of the simple transformation in Lemma 3.1 turns out to be very essential in order to reduce to the special cases in Theorems 1.7 and 1.8 and to save time in practical situations. The work we need 
to do is reduced and since the solution set is very simple, we expect that it is quite effective to use in practice. The results in Section 2.5 illustrate this point.

In the same vein (and actually with more evidences as will be shown), we can also see that applying Theorem 1.7 is quite effective in practice. In fact given a matrix $A$ and a special value $z=Z_{k}$, the system we need to check for Conjecture NC consists of only $n$ equations in $n$ variables $y_{1}, \ldots, y_{n}$. The solution set we look for is the simplest possible, that is the empty set. Moreover, a generic Druzkowski matrix satisfies Conjecture NC automatically, as shown in Theorem 1.8. When $k<n$, the proof of Theorem 1.7 shows that we can consider only special values $y$ for which $y_{k+1}^{2}=y_{k}, \ldots, y_{n}^{2}=y_{n}$, thus the mentioned system becomes one of $n$ equations in only $k$ variables $y_{1}, \ldots, y_{k}$ and hence is expected to have no solutions because of the dimension reason. All these points come together to help us to solve these systems very quickly in practice, as illustrated in Section 2.5.

Here we note one other feature, that of the generality of the approaches. Most of the existing approaches are for Keller maps only, because they consist in constructing inverse maps. So while these approaches are useful in that they not only check that a given Keller map has an inverse but also construct that inverse, they are not intended for maps which are not invertible. The Mathieu subspaces approach in the paper [12] is one of the rare generalisations of the Jacobian conjecture which still holds until now. Our approaches here point out some more possible generalisations of the Jacobian conjecture: one is that Condition C1 is true for all Druzkowski matrices, one other is that Conditions $\mathrm{C} 1$ and $\mathrm{C} 2$ are true for all matrices with integer coefficients, and yet another one is that $W_{n}$ is connected. The authors of [12] show that the conditions in their paper are satisfied by many polynomial maps which are not invertible. Likewise, here we show that Conditions C1 and C2 are true for many matrices which are not Druzkowski. Moreover, we show that Conditions C1 and $\mathrm{C} 2$ are true for a dense set of square matrices, and this enables us to propose a heuristic argument for the truth of the Jacobian conjecture. However, the Mathieu subspaces approach in [12] is probabilistic in nature and their conditions involve all polynomials and a parameter $m$ to run all over $\mathbb{N}$, hence seems not easy to employ computer programs to study (and there is no such attempt as far as we know). In contrast, our approach is algebraic in nature, and hence is very easy to employ computer programs.

\subsection{Approach 0: Checking Conjecture NC with a generic Druzkowski map}

This is the approach that we find most practical and is based on the arguments in Section 5.1. The idea is as follows. As pointed out in Section 5.1, a generic Druzkowski map satisfies the criteria in Theorem 1.8, hence we expect to be able to check very quickly that the Groebner Basis for the system $Z_{k}+A \cdot\left(Z_{k}^{3}+Z_{k} *(A . y)^{2}\right)=$ 0 (or even the stronger system $Z_{k}+A .\left(Z_{k}^{3}+Z_{k} * y^{2}\right)=0$ ) is $\{1\}$. Then, we will be able to write 1 in terms of the $n$ polynomials defining the system. If we have done 
this for a big enough number of Druzkowski matrices in a certain dimension $n$, we will be able to write down (using extrapolation) 1 in terms of the $n$ polynomial for a general Druzkowski matrix of that given dimension $n$.

The difficulty here is that we do not know much about Druzkowski matrices in higher dimensions. However, we do not really need to know all Druzkowski matrices in a certain dimension. What we really need is to be able to generate a (finite) "good" set of such Druzkowski matrices. Then, for each of Druzkowski matrices in this finite set we expect to be able to check very quickly. This is very promising, for example we can generate many such maps by starting from some classes of polynomial automorphisms (there are many good such ones, for example the tame class) and then going through the procedures given by Bass, Connell, Wright, Yagzhev and Druzkowsk to produce Druzkowski matrices.

\subsection{Approach 1: Proving inductively on $k$}

From Theorem 1.7, there is also one approach that we can utilise that is of inductively proving on $k$. This approach seems to be quite natural. Right now we know that for $k=1$ or 2 , the approach works. The proofs in these cases show that for these small values of $k$, only a small portion of the polynomials defining the Druzkowski matrices is needed (more precisely, only polynomials of total degrees 1 and 2 are needed). Hence, we expect that to check Conjecture NC for a certain value $k$, only polynomials of total degrees up to $k$ are needed. Then, we can use computer programs to check Conjecture $\mathrm{NC}$ for small values of $k$, and to deduce an inductive argument based on the observed patterns.

We note that commonly, the approach used by other authors is to restrict the ranks of $A$. However, the results proven using this approach all reduce to showing that if the rank of $A$ is very small $(1,2,3)$ or very large $n-1, n-2, n-3$, then $A$ is "equivalent" to a matrix of a very special type. It is not expected that for other values of the rank, the matrix $A$ is still "equivalent" to matrices of these types. So it is not clear how to proceed with this approach for other values of rank $A$.

\subsection{Some other approaches}

Here we mention some other possible approaches.

- Approach 2: Checking Conditions $\mathrm{C} 1$ and $\mathrm{C} 2$ on a larger variety. For Druzkowski matrices, Condition C2 is equivalent to the Jacobian conjecture. From the experimental computations above, it seems that the stronger Condition $\mathrm{C} 1$ may be also true for Druzkowski matrices. We also know that Condition C1 is true for many other varieties. So if we assume that the Jacobian conjecture is true, then there are varieties strictly containing the Druzkowski matrices on which Condition C2 (and may be also Condition C1) holds. If we can find a "nice" such variety (for example if it is defined by less equations), then we can facilitate the use of computer programs. To guess what should be such nice varieties, we may again do experiments 
with small dimensions or specific matrices. The ideal case is that Condition C2 is true for all matrices. See the Remark in Section 5.1 for support to this approach.

- Approach 3: Checking Conditions C1 and C2 for integer matrices. Here is another approach, which we feel is also promising. We know that it is sufficient to check either Condition $\mathrm{C} 1$ or $\mathrm{C} 2$ is true for all integer matrices. This is in general a hard problem belonging to the field of Diophantine equations, but we may go around it in the following way. We can compute the projections from $V_{n} \cap\left\{z=Z_{k}\right\}$ (or $W_{n} \cap\left\{z=Z_{k}\right\}$ ) to the set of all $n \times n$ matrices $\mathcal{M}_{n}$. Since this image is contained in a strictly smaller subvariety of $\mathcal{M}_{n}$ (Theorem 1.5), the closure of the image is also contained in the same strictly smaller subvariety. The ideal defining the latter (which then must be bigger than 0) can be computed explicitly ( [11]), and we can look to see whether it contains some special polynomials $P$ which is always nonzero on rational numbers (in particular, if it is always positive on real numbers). (More generally, we can check whether there is a polynomial $P$ whose zero set is easy to study.) We have searched on randomly generated matrices of various dimensions and ranks, and did not yet find any counterexample to this approach.

- Approach 4: Checking that $W_{n}^{\prime}$ or $W_{n}$ is connected. This approach is based on Theorem 1.6, which says that $J C(\infty)$ is equivalent to the fact that $W_{n}^{\prime}$ is connected for all $n \in \mathbb{N}$. In particular, if $W_{n}$ is connected for all $n \in \mathbb{N}$ then $J C(\infty)$ follows. Connectedness of a variety is a classical subject in Algebraic Geometry, and in principle it is easier to check whether a variety is connected than to compute the Groebner Basis of the ideal defining it. The fact that $W_{n}$ is defined by only $n+n^{2}$ equations makes the computations faster.

- Approach 5: Numerical methods. We can use numerical methods and ideas behind them to help resolving Conjecture NC. One such method is the homotopy continuation, which proposes to study a homotopy between a system of polynomial equations $S_{1}$ and another simpler system of polynomial equations $S_{2}$, which we expect to have the same number of solutions. In Conjecture NC, we expect that the systems $Z_{k}+A .\left(Z_{k}^{3}\right)+A .\left(Z_{k} *(A . y)^{2}\right)=0$ have no solutions for any $k=1, \ldots, n$. Hence, if $S_{1}$ is the system in Conjecture NC with some value $k$, we can choose $S_{2}$ any system without solutions. In particular, we may choose $S_{2}$ to be the system in Conjecture $\mathrm{NC}$ with a smaller value of $k$ (e.g. 1 or 2), for which we already knew the answer. This can be also done numerically by using computer programs on which these methods are already installed.

\subsection{A related problem}

To prove $J C(\infty)$ it suffices to prove for generic Druzkowski matrices. It is hence an important task to be able to generate more Druzkowski matrices so we can test the approaches. If we are able to do so, we can use Theorems 1.7 to effectively check. 


\section{Acknowledgements}

We are benefited from discussions with Neeraj Kashyap and Hang Tien Nguyen on computational aspects. The latter also helped with running some examples. Laughlin Andrew Campbell brought the paper [9] and some other information to our attention. The comments and suggestions of them and Finnur Larusson, Eric Bedford and Tien-Cuong Dinh helped to improve the paper. Most of the experimental computations that require long time and big memory have been done on the BigMem cluster of the Tizzard super computer of eResearch South Australia, and Andrew Hill there generously helped us. Part of the research was carried out while the author was at the Korea Institute for Advanced Study (KIAS), and we were helped by the KIAS Center for Advanced Computation (with computer resources) and Hoyoung Kim (with technical issues). John Dixon and Craig Bauling from the Wolfram company replied to our enquiries, in particular instructed us to a Mathematica command which describes the Groebner Basis in terms of the original defining polynomials. We would like to thank all these institutions and people for their valuable help. We thank the referee for many detailed and helpful comments and suggestions. This work has been partially supported by research grants DP120104110 and DP150103442 from Australian Research Council, and Young Research Talents grant 300814 from Research Council of Norway, as well as travel fund from the Trond Mohn Foundation.

\section{References}

[1] E. Adamus, P. Bogdan and Z. Hajto, An effective approach to Picard-Vessiot theory and the Jacobian conjecture, Schedae Informaticae, 2017, Volume 26, 49-60.

[2] E. Adamus, P. Bogdan and Z. Hajto, Example of a Druzkowski map, Link: http://crypto.ii.uj.edu.pl/galois/

[3] The arXiv page on the Jacobian conjecture: https://en.wikipedia.org/wiki/Jacobian_conjecture

[4] M. de Bondt, Homogeneous Keller maps, PhD dissertation, Radboud University Nijmegen, 2009. Link: http://www.math.ru.nl/ debondt/promotion.html

[5] M. de Bondt and A. van den Essen, The Jacobian conjecture for symmetric Druzkowski mappings, Ann. Polon. Math. 86 (2005), no 1, 43-46.

[6] M. de Bondt and D. Yan, Some remarks on the Jacobian conjecture and Druzkowski mappings, J. Algebra 384 (2013), 267-275.

[7] M. de Bondt and D. Yan, Some remarks on the Jacobian conjecture and polynomial endomorphisms, Proc. AMS 142 (2014), no 2, 391-400.

[8] M. de Bondt and D. Yan, Triangularization properties of power linear maps and the structural conjecture, Ann. Polon. Math. 112 (2014), no 3, 247-266.

[9] A. Cima, A. Gasull, J. Llibre and F. Manosas, Global injectivity of polynomial maps via vector fields, In "Automorphisms of affine spaces" (Curacao, 1994), 105-123, Kluwer Academia Publishers, Dordrecht, 1995.

[10] D. Cox, J. Little and D. O'Shea, Using algebraic geometry, Graduate texts in mathematics 185, 2nd edition, Springer Science+Business Media, Inc., 2005.

[11] D. Cox, J. Little and D. O'Shea, Ideals, varieties and algorithms, 2nd edition, Springer-Verlag New York, 1997 . 
[12] H. Derksen, A. van den Essen and W. Zhao, The Gaussian moments conjecture and the Jacobian conjecture, Israel J. Math., 219, 917-928.

[13] L. Druzkowski, The Jacobian conjecture: symmetric reduction and solution in the symmetric cubic linear case, Ann. Polon. Math. 87 (2005), 83-92.

[14] L. Druzkowski, New reduction in the Jacobian conjecture, English summary, Effective methods in algebraic and analytic geometry (Krakow). Univ. Iagel. Acta Math. No. 39 (2001), 203-206.

[15] L. Druzkowski, The Jacobian conjecture in case of rank or corank less than three, J. Pure Appl. Algebra 85 (1993), 233-244.

[16] L. Druzkowski and K. Rusek, The formal inverse and the Jacobian Conjecture, Ann. Polon. Math. 46 (1985), 85-90.

[17] A. van den Essen, To believe or not to believe: The Jacobian conjecture, Rend. Sem. Mat. Univ. Pol. Torino, Vol. 55, 4 (1997), Jacobian Conj. and Dyn. Syst.

[18] D. Eisenbud and E. G. Evans, Every algebraic set in $n$-space is the intersection of $n$ hyper surfaces, Invent. Math. 19, 107-112 (1973).

[19] A. van den Essen, Polynomial automorphisms and the Jacobian conjecture, Birkhauser Verlag, 2000.

[20] G. Gorni and G. Zampieri, On cubic-linear polynomial mappings, Indag. Math., N.S., 8 (4), 471-492 (1997).

[21] G. Gorni, H. Tutaj-Gasinska and G. Zampieri, Druzkowski matrix search and D-nilpotent automorphisms, Indag. Math., N.S., 10 (2), 235-245 (1999).

[22] E. Hubbers, Cubic similarity in dimension five, Link http://www.math.unl.edu/events/special/meisters/papers/hubbers.pdf

[23] J. E. Humphreys, Conjugacy classes in semisimple algebraic groups, Mathematical surveys and monographs, Volume 43, American Mathematical Society, Providence, Rhode Island, 1995.

[24] G. Meisters, Wanted: A bad matrix, The American Mathematical Monthly, Vol 102, no 6 (June - July 1995), 546-550.

[25] G. Meisters, Invariants of cubic similarity, Link:

http://www.math.unl.edu/ gmeisters1/papers/TrentoPaper/invariantsCS.pdf

[26] K. Rusek, A geometric approach to Keller's Jacobian conjecture, Math. Ann. 264, 315-320 (1983).

[27] https://arxiv.org/pdf/1503.08733.pdf

[28] The Wikipedia page on the Jacobian conjecture.

[29] A. V. Yaghzev, On Keller's problem, Siberian Math. J. 21 (1980), 141-150.

[30] D. Yan, A note on the Jacobian conjecture, Linear Algebra Appl. 435 (2011), no $9,2110-2113$. 